\title{
Theoretic and Numerical Study of a New Chaotic System
}

\author{
Congxu Zhu, Yuehua Liu, Ying Guo \\ School of Information Science and Engineering, Central South University, Changsha 410083, China \\ Email: zhucongxu@126.com
}

\begin{abstract}
This paper introduced a new three-dimensional continuous quadratic autonomous chaotic system, modified from the Lorenz system, in which each equation contains a single quadratic cross-product term, which is different from the Lorenz system and other existing systems. Basic properties of the new system are analyzed by means of Lyapunov exponent spectrum, Poincaré mapping, fractal dimension, power spectrum and chaotic behaviors. Furthermore, the forming mechanism of its compound structure obtained by merging together two simple attractors after performing one mirror operation has been investigated by detailed numerical as well as theoretical analysis. Analysis results show that this system has complex dynamics with some interesting characteristics.
\end{abstract}

Keywords: Chaotic System, Lyapunov Exponent, Poincaré Mapping, Fractal Dimension, Power Spectrum

\section{Introduction}

Chaos is found to be useful or has great potential application in many disciplines. Recently, it has been noticed that purposefully creating chaos can be a key issue in many technological applications such as communication, encryption, information storage, etc. Since Lorenz found the first chaotic attractor in a three-dimensional (3-D) autonomous system in 1963 [1], Lorenz system has become a paradigm for chaos research, and many new Lorenz-like chaos system have been constructed [2-6]. In finding of a new system, one can construct and determine the system parameter values such that the system can become chaotic following some basic ideas of chaotification [7], namely:

1) It is dissipative.

2) There exist some unstable equilibria, especially some saddle points.

3) There are some cross-product terms, so there are dynamical influence between different variables.

4) The system orbits are all bounded.

This motivates the present study on the problem of generating new chaotic attractors. Under these guidelines, though not sufficient, a new chaotic system is generated by modifying from the Lorenz system. It turns out that the new system has five equilibria, therefore is not topologically equivalent to the Lorenz system. This paper has also briefly studied and analyzed its forming mechanism. The compound structure of the attractor obtained by merging together two simple attractors after performing one mirror operation is explored here. Simulation results support brief theoretical derivations. The proposed approach in finding a new chaotic system has advantages of intuitiveness, simpleness, and convenience over some existing methods. But it has a shortcoming of uncertainty.

In the rest of the paper, we use the lowercase letters $x$, $y, z$ denote the state variables of the new $3 \mathrm{D}$ chaotic system, the uppercase letter $\mathrm{X}$ denotes the vector of state variables, $F$ denotes the name of function, $\mathrm{J}$ denotes the Jacobian matrix, $\lambda$ denotes the eigenvalue of a matrix, $L$ denotes the Lyapunov exponents of the system.

\section{The New Chaotic System and Its Properties}

The new chaotic system introduced in this paper is described as the following autonomy differential equations:

$$
\left\{\begin{array}{l}
\dot{x}=-x-a y+y z, \\
\dot{y}=b y-x z \\
\dot{z}=-c z+x y .
\end{array}\right.
$$

Here, $a, b, c$ are constant parameters of the system. When $a=1.5 ; b=2.5 ; c=4.9$, system (1) is chaotic and belongs Lorenz system family. In system (1) each equation contains a single quadratic cross-product term, and the linear terms in the first and second equations are dif- 
ferent from the Lorenz system, and therefore is a new Lorenz-like attractor, but not equivalent chaotic attractor in the topological structure [1].

\subsection{Symmetry and Dissipativity}

System (1) has a natural symmetry under the coordinates transform $(x, y, z) \rightarrow(-x,-y, z)$, which persists for all values of the system parameters. So, system (1) has symmetry about the $z$-axis.

system (1) can be expressed as the form of vectors

$$
\dot{X}=\boldsymbol{F}(X)=\left[f_{1}(X), f_{2}(X), f_{3}(X)\right]^{\mathrm{T}} .
$$

where $X=[x(t), y(t), z(t)]^{\mathrm{T}}$, The divergence of the vector field $F(X)$ on $\mathbf{R}^{3}$ is given by

$$
\nabla \boldsymbol{F}=\frac{\partial f_{1}(X)}{\partial x}+\frac{\partial f_{2}(X)}{\partial y}+\frac{\partial f_{3}(X)}{\partial z}
$$

The divergence of $F$ measures how fast volumes change under the flow $\Phi_{t}$ of $F$. Suppose $D$ is a region in $\mathbf{R}^{3}$ with a smooth boundary, and let $D(t)=\Phi_{t}(D)$, the image of $D$ under the time $t$ map of the flow. Let $V(t)$ be the volume of $D(t)$. Then Liouville's theorem asserts that

$$
\frac{\mathrm{d} V(t)}{\mathrm{d} t}=\int_{D(t)}(\nabla \boldsymbol{F}) \mathrm{d} x \mathrm{~d} y \mathrm{~d} z
$$

For the system (1), we compute immediately that the divergence is the negative constant $-(c+1-b)=-3.4$, so that volume decreases at a constant rate

$$
\frac{\mathrm{d} V(t)}{\mathrm{d} t}=-(c+1-b) \int_{D(t)} \mathrm{d} x \mathrm{~d} y \mathrm{~d} z=-(c+1-b) V
$$

Solving this simple differential Equation (5) yields

$$
V(t)=V(0) e^{-(c+1-b) t}=V(0) e^{-3.4 t}
$$

So that any volume must shrink exponentially fast to 0 , and dynamical system described by (1) is a dissipative system. Hence, all orbits of system (1) are eventually confined to a specific subset that have zero volume, the asymptotic motion settles onto an attractor of the system (1) $[8]$.

\subsection{System Equilibria}

In this section, assume that $b \neq 0, b c>0$, and $a^{2}+4 b>0$. The equilibria of system (1) are found by solving the three equations $\dot{x}=\dot{y}=\dot{z}=0$. It is found that system (1) has five equilibria, which are respectively described as follows:

$O(0,0,0) ; E_{1}\left(x_{0}, y_{+}, z_{+}\right) ; E_{2}\left(x_{0}, y_{-}, z_{-}\right) ; E_{3}\left(-x_{0},-y_{-}, z_{-}\right) ;$ and $E_{4}\left(-x_{0},-y_{+}, z_{+}\right)$, where $x_{0}=\sqrt{b c}$,

$$
\begin{aligned}
& y_{+}=\sqrt{b c}\left(a+\sqrt{a^{2}+4 b}\right) /(2 \mathrm{~b}), \\
& y_{-}=\sqrt{b c}\left(a-\sqrt{a^{2}+4 b}\right) /(2 \mathrm{~b}), \quad z_{+}=\left(a+\sqrt{a^{2}+4 b}\right) / 2,
\end{aligned}
$$

and $z_{-}=\left(a-\sqrt{a^{2}+4 b}\right) / 2$. When $a=1.5 ; b=2.5 ; c=4.9$, the five equilibria are $O(0,0,0) ; \quad E_{1}(3.5,3.5,2.5)$; $E_{2}(3.5,-1.4,-1) ; E_{3}(-3.5,1.4,-1)$; and $E_{4}(-3.5,-3.5,2.5)$.

The Jacobian matrix $J$ of system (1) is

$$
\boldsymbol{J}=\left[\begin{array}{lll}
\frac{\partial f_{1}(X)}{\partial x} & \frac{\partial f_{1}(X)}{\partial y} & \frac{\partial f_{1}(X)}{\partial z} \\
\frac{\partial f_{2}(X)}{\partial x} & \frac{\partial f_{2}(X)}{\partial y} & \frac{\partial f_{2}(X)}{\partial z} \\
\frac{\partial f_{3}(X)}{\partial x} & \frac{\partial f_{3}(X)}{\partial y} & \frac{\partial f_{3}(X)}{\partial z}
\end{array}\right]=\left[\begin{array}{ccc}
-1 & z-a & y \\
-z & b & -x \\
y & x & -c
\end{array}\right] \text { (7) }
$$

For equilibrium point $O(0,0,0)$, the Jacobian matrix has the following result:

$$
\boldsymbol{J}_{0}=\left[\begin{array}{ccc}
-1 & -1.5 & 0 \\
0 & 2.5 & 0 \\
0 & 0 & -4.9
\end{array}\right]
$$

To gain its eigenvalues, let $\left[\lambda \boldsymbol{I}-\boldsymbol{J}_{0}\right]=0$, Then the eigenvalues that corresponding to equilibrium $O(0,0,0)$ are respectively obtained as follows: $\lambda_{1}=-4.9, \lambda_{2}=2.5, \lambda_{3}=-1$. Here $\lambda_{2}$ is a positive real number, $\lambda_{1}$ and $\lambda_{3}$ are two negative real numbers. Therefore, the equilibrium $O(0,0,0)$ is a saddle point. So, this equilibrium point $O(0,0,0)$ is unstable.

In the same way, the eigenvalues that corresponding to equilibrium point $E_{1}$ are obtained as: $\lambda_{1}=-6.5337$, $\lambda_{2}=1.5+3.2664 i, \lambda_{3}=1.5-3.2664 i$, where $i$ denote the unit of imaginary number. The eigenvalues that corresponding to equilibrium point $E_{2}$ are obtained as: $\lambda_{1}=-4.4632$, $\lambda_{2}=0.5316+2.7208 i, \lambda_{3}=0.5316-2.7208 i$. The eigenvalues that corresponding to equilibrium point $E_{3}$ and $E_{2}$ are the same, and the eigenvalues that corresponding to equilibrium point $E_{4}$ and $E_{1}$ are also the same.

For each of the four equilibrium points $E_{1}, E_{2}, E_{3}$ and $E_{4}$, the results show that $\lambda_{1}$ is a negative real number, $\lambda_{2}$ and $\lambda_{3}$ become a pair of complex conjugate eigenvalues with positive real parts. Therefore, equilibrium points $E_{1}$, $E_{2}, E_{3}$ and $E_{4}$ are all saddle-focus points; so, these equilibrium points are all unstable.

\subsection{Observation of Chaotic and Complex Dynamics}

The initial values of the system are selected as $(-0.5,2.0$, 3.5). Using MATLAB program, the numerical simulation have been completed. This nonlinear system exhibits the complex and abundant chaotic dynamics behaviors, the strange attractors are shown in Figures 1. Apparently, the strange attractors in this nonlinear-system are different 
from Lorenz chaos attractor.

The waveforms of $x(t)$ in time domain are shown in Figure 2. The waveforms of $x(t)$ is aperiodic.

In order to discriminate between a multiple periodic
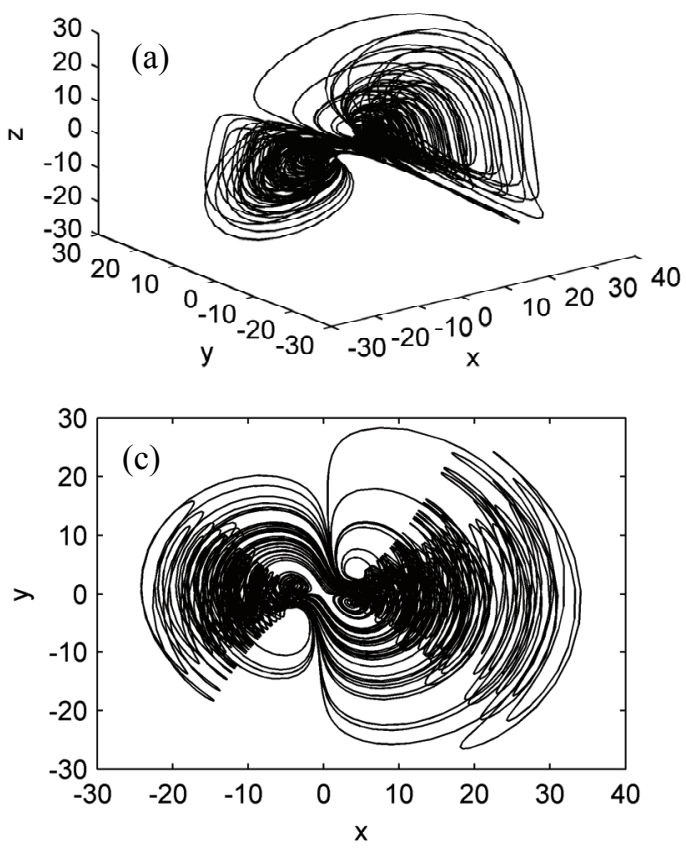

motion that can show also a complicated behavior and a chaotic motion, the power spectrum of nonlinear system (1) is also studied, and it is continuous as shown in Figure 3. The Poincaré section for chaotic attractors shows a certa-
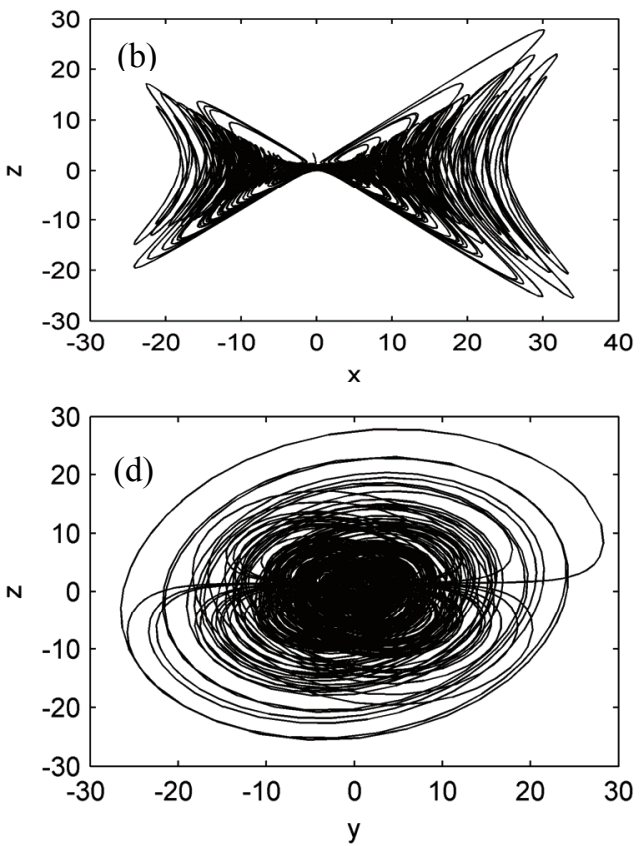

Figure 1. Phase plane strange attractors. (a) Three- dimensional view; (b) $x-z$ phase plane; (c) $x-y$ phase plane; (d) $y-z$ phase plane

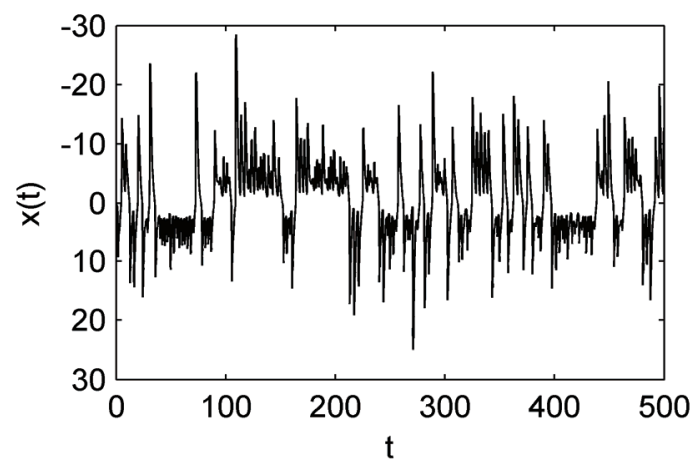

Figure 2. $x(t)$ waveform of system (1)

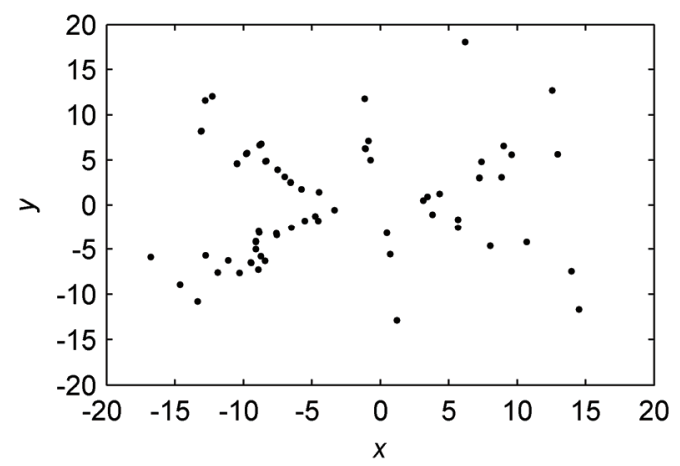

Figure 4. The Poincaré map of $x-y$ plane

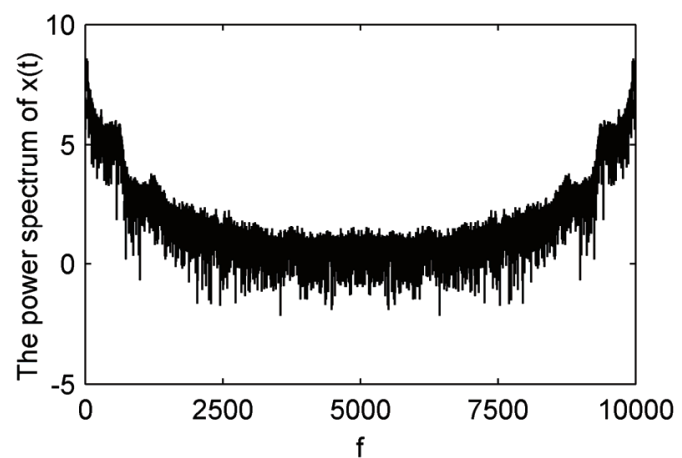

Figure 3. Power spectrum of $x(t)$

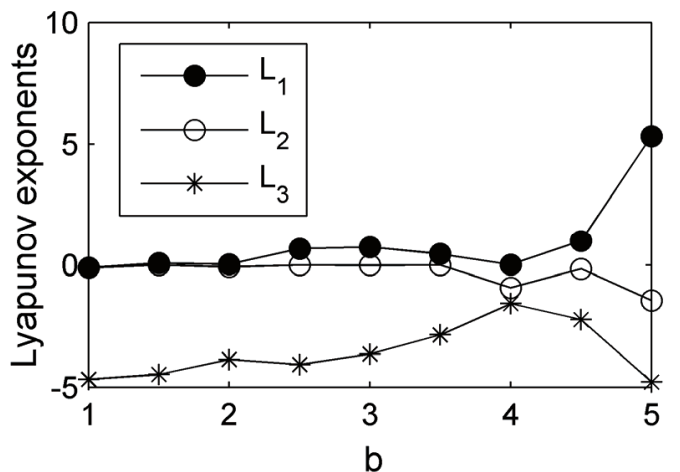

Figure 5. Spectrum of Lyapunov exponents 
in type of organization but with a complex geometry. The section can be composed by an infinity of points (in contrast with the finite number of points for the periodic and quasi-periodic case) irregularly scattered, in one line or many arks of curves. Poincaré mapping of system (1) is shown in Figure 4, its structure becomes better and better defined in time after the accumulation of points.

As is well known, the Lyapunov exponents measure the exponential rates of divergence or convergence of nearby trajectories in phase space, according to the detailed numerical as well as theoretical analysis, the largest value of positive Lyapunov exponents of this chaotic system is obtained as $L_{1}=0.6747$. It is related to the expanding nature of different direction in phase space.

Another one Lyapunov exponent is $L_{2}=0$. It is related to the critical nature between the expanding and the contracting nature of different direction in phase space.

While negative Lyapunov exponent is $L_{3}=-4.0738$. It is related to the contracting nature of different direction in phase space.

Fix parameters $a=1.5, c=4.9$, and let $b$ vary. The spectrum of Lyapunov exponents of system (1) versus $b$ are computed, and the results are shown in Figure 5.

The Lyapunov dimension of chaos attractors of this nonlinear system is of fraction dimension, it is described as

$$
D_{L}=j+\frac{1}{\left|L_{j+1}\right|} \sum_{i=1}^{j} L_{i}=2.166
$$

The fractal nature of an attractor does not merely imply non-periodic orbits; it also causes nearby trajectories to diverge. As all strange attractors, orbits that are initiated from different initial conditions soon reach the attracting set, but two nearby orbits do not stay close to each other [9]. They soon diverge and follow totally different paths in the attractor. Therefore, there is really chaos in this nonlinear system.

\section{Forming Mechanism of This New Chaotic Attractor Structure}

In order to reveal the forming mechanism of this new chaotic attractor structure, its controlled system is proposed, the autonomous differential equations of its controlled system are expressed as

$$
\begin{aligned}
& \dot{x}=-x-1.5 y+y z, \dot{y}=2.5 y- \\
& x z+k, \dot{z}=-4.9 z+x y .
\end{aligned}
$$

In this system, $k$ is the parameter of control, the value of it can be changed within a certain range.

When the parameter $k$ is changed, the chaos behavior of this system can effectively be controlled. So it is a controller. Here, the initial values of the system are selected as $(-0.5,0,0.5)$.
Let $k=1.9$, the corresponding strange attractors are shown in Figure 6(a).the attractor evolves into partial but is still bounded in this time.

Let $k=2.1$, the corresponding strange attractors are shown in Figure 6(b). Moreover the strange attractors are evolved into single left scroll attractor; it is only one half the original chaotic attractors in this time.

Let $k=2.5$, the strange attractor evolves into the period-doubling bifurcations; period-doubling bifurcations are shown in Figure 6(c).

While $k$ is a negative value, the chaos of this system can also be affected. The initial values of the system are still selected as $(-0.5,0,0.5)$.

Let $k=-1.9$, the strange attractors are shown in Figure 6(d), the attractor evolves also into partial but is still bounded in this time.

Let $k=-2.1$, the corresponding strange attractors are shown in Figure 6(e). Moreover the strange attractors are evolved into single right scroll attractor; it is also only one half the original chaotic attractors in this time.

Let $k=-2.5$, the strange attractor evolves into the period-doubling bifurcations; period-doubling bifurcations are shown in Figure 6(f).

In the controller, one can see when $|k|$ is large enough, chaos attractor disappears; when $|k|$ is small enough, a complete chaos attractor appears. So $|k|$ is an important parameter to control chaos in the nonlinear-system [10].

This means the attractor is a compound structure obtained by merging together two simple attractor after performing one mirror operation [11].

\section{Conclusions}

This paper has reported and analyzed a new three- dimensional continuous autonomous chaotic system, in which each equation has a single cross-product term. Basic properties of the system have been analyzed by means of Lyapunov exponents, Poincaré mapping, fractal dimension, power spectrum and chaotic behaviors. This new attractors proposed can be also realized with an electronic circuit and have great potential for communication and electronics [12]. Apparently there are more interesting problems about this new system in terms of dynamics, complexity, control and synchronization, among others, leaving rooms for further studies.

\section{Acknowledgments}

This work was supported by the National Natural Science Foundation of China under Grant 60902044 and the First Batch Fostering Foundation in Training Graduate Students of Central South University. 

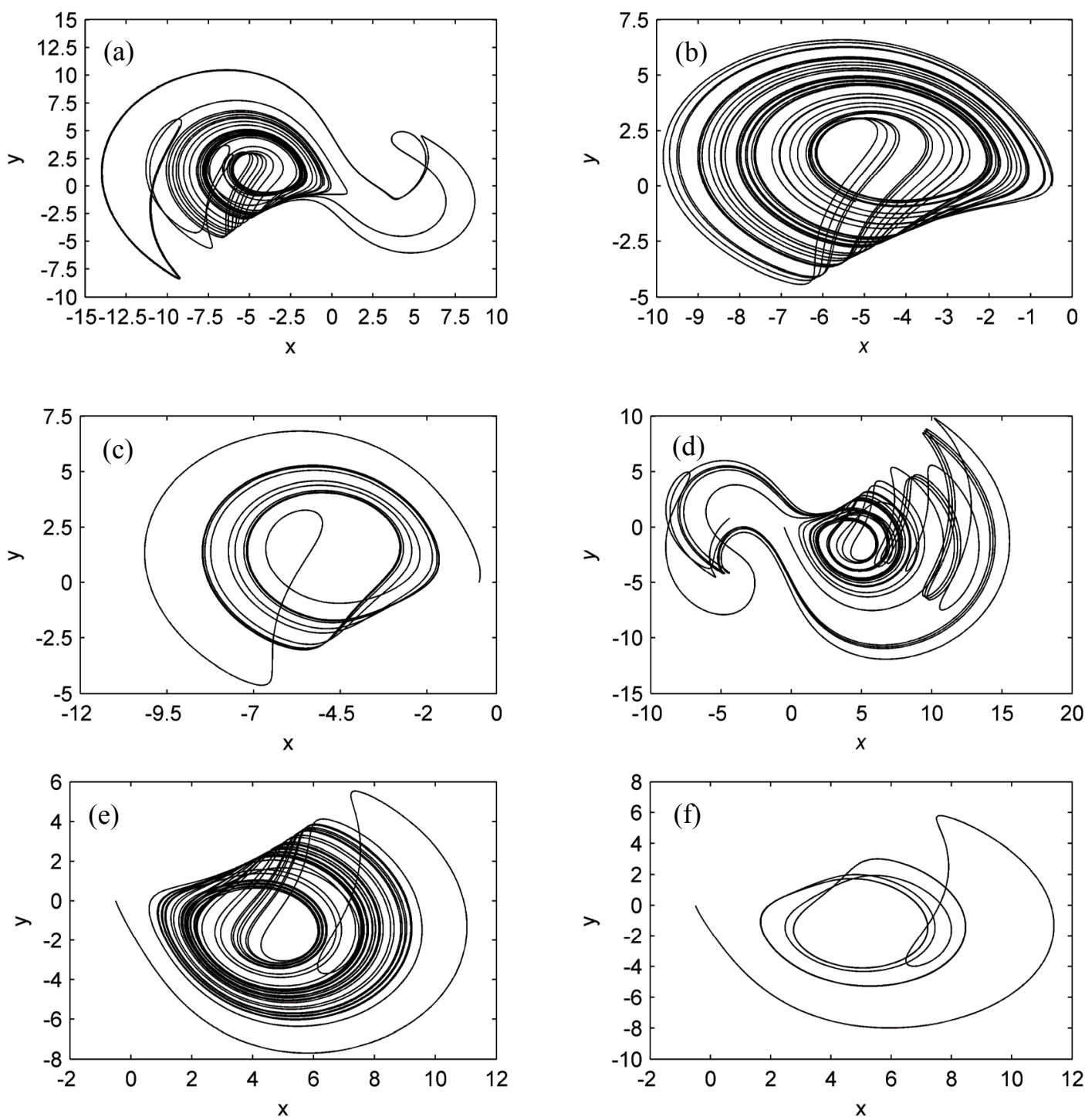

Figure 6. Dynamics behavior of the system changs with the control parameter $k$. a) $x-y$ phase plane strange attractors $(k=1.9)$; b) $x-y$ phase plane strange attractors $(k=2.1)$; c) $x$-y phase plane period-doubling bifurcations $(\mathrm{k}=2.5)$; d) $x$-y phase plane strange attractors $(k=-1.9)$; e) $x-y$ phase plane strange attractors $(k=-2.1)$; $) x-y$ phase plane period-doubling bifurcations $(k=-2.5)$.

\section{References}

[1] C. Sparrow, "The Lorenz equations: Bifurcations chaos and strange attractors," Springer, New York, 1982.

[2] G. R. Chen and T. Ueta, "Yet another chaotic attractor," International Journal of Bifurcation and Chaos, Vol. 9, No. 7, pp. 1465-1466, 1999.

[3] J. H. Lü and G. R. Chen, "A new chaotic attractor coined," International journal of bifurcation and chaos, Vol. 12, No. 3, pp. 659-661, 2002.

[4] J. H. Lü, G. R. Chen, D. Cheng, and S. Celikovsky, "Bridge the gap between the Lorenz system and the Chen system," International Journal of Bifurcation and Chaos, Vol. 12, No. 12, pp. 2917-2926, 2002.

[5] G. Qi, G. R. Chen, and S. Du, "Analysis of a new chaotic system," Physica A, Vol. 352, No. 2-4, pp. 295-308, 2005

[6] C. X. Liu, L. Liu, and T. Liu, "A new butterfly-shaped attractor of Lorenz-like system," Chaos, Solitons \& Fractals, Vol. 28, No. 5, pp. 1196-1203, 2006.

[7] X. Wang, "Chaos control," Springer, New York, 2003.

[8] J. H. Lü, G. R.Chen, and S. Zhang, "Dynamical analysis of a new chaotic attractor," International Journal of Bifurcation and Chaos, Vol. 12, No. 5, pp. 1001-1015, 2002. 
[9] A. Wolf, J. B. Swift, H. L. Swinney, and J. A. Vastano, "Determining Lyapunov exponents from a time series," Physica D, Vol. 16, No. 3, pp. 285-317, 1985.

[10] C. X. Liu, L. Liu, and K. Liu, "A new chaotic attractor," Chaos, Solitons \& Fractals, Vol. 22, No. 5, pp. 10311038, 2004.

[11] J. H. Lü, G. R. Chen, and S. Zhang, "The compound structure of a new chaotic attractor," Chaos, Solitons \& Fractals, Vol. 14, No. 5, pp. 669-672, 2002.

[12] G. Q. Zhong and W. K. S. Tang, "Circuitry implementation and synchronization of Chen's attractor," International Journal of Bifurcation and Chaos, Vol. 12, No. 6, pp. $1423-1427,2002$. 